\title{
EL MÉTODO CIENTÍFICO DE GALILEO Y SUS IMPLICANCIAS CULTURALES
}

\author{
The Scientific Method of Galileo and its Cultural Implications
}

Paolo Musso*

\begin{abstract}
RESUMEN
El presente artículo presenta las contribuciones de Galileo a la ciencia moderna, en especial el de establecer de manera definitiva el método de la ciencia natural, donde no sólo la observación es parte de este conocimiento sino también la experimentación, el no usar la esencia de las cosas, sino limitarse a estudiar algunas propiedades, el uso de las matemáticas, etc. Se indica además que contribuyeron factores culturales que favorecieron el proceso científico de esta época como la fe griega y cristiana en el orden racional de mundo, el redescubrimiento del platonismo y del texto griego matemáticos y finalmente la fe cristiana en la creación del mundo.
\end{abstract}

\section{Palabras clave}

Método científico, Galileo, revolución científica, experimentación, matemáticas, fe griega y fe cristiana

\begin{abstract}
This article presents the contributions of Galileo to modern science, especially that of definitively establishing the method of natural science, where not only observation is part of this knowledge but also experimentation, not using the essence of the things, but simply to study some properties, the use of mathematics, etc. It also indicates that cultural factors that favored the scientific process of this time contributed as the Greek and Christian faith in the rational order of the world, the rediscovery of Platonism and the Greek text mathematicians and finally the Christian faith in the creation of the world.
\end{abstract}

\section{Keywords:}

Scientific method, Galileo, scientific revolution, experimentation, mathematics, Greek faith and Christian faith

* Docente de la "Universitá degli Studi dell'Insubria di Varese - Italia: paolo.musso@ucss.edu.pe 


\section{El padre de la ciencia moderna}

De hecho, la revolución científica ya había empezado antes de Galileo.

Y entonces, ¿por qué decimos igualmente que el verdadero padre de la ciencia moderna es Galileo?

Está claro que en cualquier discurso de este tipo siempre hay algo convencional, porque ninguno empieza realmente "solo" una revolución de este alcance: siempre se necesita una larga preparación, que involucra a muchas personas y puede durar hasta siglos, como de hecho aconteció. Sin embargo, es también verdad que en toda revolución siempre llega, tarde o temprano, una persona o un grupo de personas (generalmente bastante pequeño) que se encarga de unificar todos dichos fermentos en una síntesis nueva y sobre todo consciente de las razones de su propia novedad, que al principio usualmente son percibidas de una manera bastante confusa, hasta por sus mismos protagonistas: y no cabe duda de que en el caso de la revolución científica del Renacimiento quien hizo esto fue el propio Galileo. En efecto, él dio 3 contribuciones de importancia transcendental, cada una de las cuales ya habría sido suficiente como para pasar a la historia: claro que las tres juntas justifican ampliamente que se le considere como la que marcó el punto de inflexión decisivo en la historia de la ciencia natural. En efecto, Galileo por primera vez:

1) Probó la unidad de la naturaleza, demostrando, gracias a sus descubrimientos astronómicos, que, al contrario de lo que se creía antes, los cuerpos celestes no son esferas perfectas ni tampoco están hechos de una materia diferente a la de la Tierra, y que por tanto las mismas leyes naturales rigen tanto en la Tierra como en las estrellas más lejanas.

2) Descubrió las primeras leyes naturales realmente fundamentales, las del movimiento de los cuerpos, que además eran necesarias para comprobar el heliocentrismo.

3) Estableció de una manera clara y definitiva el método de la ciencia natural, en que se basan no solo sus personales descubrimientos, sino también todos los otros que se han dado después de él, a lo largo de los siglos.

Y es exactamente de este último que ahora vamos a hablar.

\section{El método de la ciencia natural}

Como hemos recién dicho, Galileo definió, de una manera extremadamente clara, simple y sobre todo definitiva, el método de la ciencia natural, que sigue siendo idéntico hoy en día, después de 4 siglos, lo que es casi increíble, más aún si pensamos en que él nunca escribió un ensayo sistemático sobre del tema, sino que sus principios metodológicos se encuentran esparcidos en varios pasajes de sus libros y a veces incluso cartas.

Pero hay más. En efecto, esto no fue solo un logro práctico, sino el descubrimiento de un nuevo modo de usar la razón. Obviamente, la razón siempre está implicada en todo lo humano, pero un nuevo método significa el descubrimiento de una nueva manera de usarla basada en reglas válidas universalmente, lo que, como se puede fácilmente entender, no acontece muy a menudo. Fue algo extraordinario, cuya importancia trasciende el ámbito de la ciencia natural. Como lo dijo Einstein, «el 
descubrimiento y el uso del razonamiento científico, por obra de Galileo, fue uno de los más importantes acontecimientos en la historia del pensamiento humano y representa el verdadero inicio de la física» (Einstein e Infeld 1938: 19).

Vemos, pues, cuáles son los 4 principios básicos del método científico según Galileo, acerca de los cuales todos los filósofos y los historiadores de la ciencia más o menos están de acuerdo (lo que acontece muy raramente y demuestra una vez más el excepcional nivel de claridad que supo alcanzar Galileo al propósito):

1) No buscar la esencia de las cosas, sino limitarse a estudiar algunas propiedades.

2) No solo genérica observación, sino experimento.

3) Uso de la matemática.

4) Ningún principio de autoridad.

Y ahora vamos a analizar cada uno en detalles.

1) Aunque obviamente todos los 4 principios tengan que ser aplicados juntos para que el método pueda funcionar, el primero es el más importante. En efecto, desde la Grecia antigua hasta Galileo todos siempre intentaron hacer la ciencia según el método deductivo, ${ }^{1}$ es decir, intentando definir por medio de la pura razón las esencias de las cosas y luego deducir de ellas todos los particulares: pero siempre fracasaron. Hay que decir que, en un sentido, los griegos fueron las víctimas de su propio éxito. En efecto, dicho método, lejos de ser bárbaro, obscurantista o supersticioso, tuvo una extraordinaria eficacia en muchas disciplinas, como la metafísica, la lógica, la matemática y, sobre todo, la geometría, gracias a los Elementos de Euclídes (siglo IV - 283 a.C.): por tanto, es del todo comprensible que los griegos y sus seguidores medioevales hubiesen pensado de usarlo también en el campo de la ciencia natural. ${ }^{2}$ Sin embargo, no todo lo que a primera vista parece justo lo es realmente.

El golpe de genio de Galileo fue exactamente el haber entendido primero que en el caso de la ciencia natural (y solo en este caso) era necesario invertir el método, empezando por los aspectos más simples y particulares, que conocemos por medio de la experiencia sensible, para después llegar a los más profundos y generales, que en cambio pueden estar (y muchas veces están realmente) más allá de la experiencia. Y esto es el famoso texto de Las manchas solares donde se encuentra la primera formulación de la inversión metodológica galileana:

Por lo tanto, o queremos intentar de penetrar especulando la esencia verdadera e intrínseca de las sustancias naturales, o queremos conformarnos con descubrir algunas propiedades suyas. Buscar la esencia lo creo un intento vano tanto

1 Muchas veces como sinónimo de "método deductivo" se usa la expresión "a priori", pero que es tomada de la obra de Kant y no es nada precisa, porque implica la idea de un sistema conceptual construido de manera completamente independiente de la experiencia sensible, según el estilo (equivocado) típico de la filosofía moderna que se empezó solo con Descartes, en el siglo XVII (Musso. cap. 2). En cambio, la ciencia aristotélica era independiente de la experiencia sensible solo en el sentido de que afirmaba que era posible llegar a conocer las esencias de las cosas a través de la pura reflexión, sin necesidad ni de cálculos ni de experimentos: sin embargo, dicha reflexión siempre era acerca de la experiencia y por esto Aristóteles en particular valoraba mucho la observación (aunque no sea la misma cosa que el experimento, como veremos de inmediato).

2 Los Elementos de Euclídes eran una obra tan moderna que su estructura axiomática será mejorada por primera vez solo después de 22 siglos, en 1899, por David Hilbert en su Grundlagen der Geometrie (Hilbert 1899). 
en el caso de las sustancias más simples como de las más lejanas y celestes: y me parece que soy igualmente ignorante sobre la sustancia de la Tierra que, de la Luna, de las nubes y de las manchas solares; ni veo que comprendiendo estas sustancias cercanas tenemos otra ventaja además del número de las propiedades, pero todas igualmente desconocidas. [...] Sin embargo, si queremos limitarnos al aprender algunas propiedades, no me parece que sea imposible descubrirlas también en los cuerpos más lejanos de nosotros, no menos que en los cercanos. (Galileo 1613: 187-188)

Es importante entender que dicha limitación solo es metodológica. Galileo no era ni un fenomenísta (es decir, uno que piensa que solo podemos conocer lo que aparece y no lo que realmente es) ${ }^{3}$ ni mucho menos un escéptico. Véase, por ejemplo, este texto, también tomado de Las manchas solares: «Los nombres y las propiedades tienen que adaptarse a la esencia de las cosas, y no la esencia a los nombres; porque antes fueron las cosas, y después los nombres) (Galileo 1613: 97). O este de los Diálogos sobre los dos máximos sistemas, hasta más contundente: «SALVIATI - Usted yerra, señor Simplicio; usted debe decir que cualquiera sabe que se llama gravedad. Pero yo no le pregunto sobre el nombre, sino sobre la esencia de las cosas»)(Galileo 1632: 260). ¿Hay contradicción? No, porque Galileo no rechazó por nada la búsqueda de la esencia, que consiste simplemente en las propiedades fundamentales de una cosa, o sea, las que hacen que sea lo que es y de las cuales por tanto derivan todas las otras propiedades suyas. Ahora bien, no cabe duda que esto es lo que la ciencia siempre ha intentado (y en parte también logrado) descubrir. Por otro lado, esto lo demuestra también el testimonio de toda su vida, pues si Galileo sufrió el proceso, fue exactamente porque nunca aceptó presentar sus ideas solo como hipótesis (lo que estaba permitido), sino como conocimientos verdaderos de las cosas, es decir, justamente, de su esencia. Sin embargo, con Galileo el conocimiento de la esencia se convierte en el punto de llegada de la investigación científica (que solo se puede lograr por grados, un poquito a la vez) en lugar de ser su punto de partida, como era para los antiguos.

Además, cabe subrayar que, aunque el método de Galileo no sea deductivo, tampoco es inductivo, como muy a menudo erróneamente se dice: en efecto, el inductivismo no es una alternativa real al deductivismo, más bien, es la otra cara de la misma moneda, ya que sigue oponiendo razón y experiencia, mientras que el método de Galileo se basa en la unidad indisoluble de las dos.

Muchos historiadores y epistemólogos no están de acuerdo conmigo y eligen a uno u otro de los demás principios como el más importante, pero en efecto hay una estrecha relación lógica entre los 4 principios galileanos, que están concatenados uno a otro de modo tal que los 3 sucesivos dependen todos, directamente o indirectamente, del primero, como ahora veremos.

2) El segundo punto del método galileano es, como hemos dicho, el

3 En efecto esta idea, hoy muy común, no solo es falsa, sino también antihistórica, pues dicha distinción entre propiedades y esencia solo nació con Kant y su famosa separación entre, exactamente, los "fenómenos" y las "cosas en sí". Según Aristóteles, en cambio, las propiedades sensibles (por lo menos las más importantes, los llamados "accidentes propios") son parte de la "esencia" de una cosa, aunque ciertamente la esencia no se reduce a un mero conjunto de propiedades sensibles (exactamente como, por otro lado, tampoco las teorías científicas se reducen a un mero conjunto de enunciados empíricos, como querían los neopositivistas). 
experimento en lugar de la simple observación. ${ }^{4}$ Ahora bien, el experimento se diferencia de la simple observación porque es artificial, orientado y repetible, lo que pide una intervención activa del científico. Pero esto solo es posible exactamente porque tiene como su tarea evidenciar y estudiar "algunas propiedades", y por tanto se basa en el primer principio.

En efecto, el experimento no se hace en la realidad, así como se da espontáneamente, sino en una situación artificial, que pone el objeto de nuestro estudio en condiciones muy particulares, cuidadosamente seleccionadas y controladas, que en la naturaleza simplemente no existen, con el fin de aislar lo más posible de las otras las propiedades que queremos estudiar, para entenderlas mejor: luego, el experimento nos obliga a hacer algo y por tanto es intrínsecamente activo. Asimismo, esto significa que el experimento siempre implica una hipótesis teórica de algún tipo, sin la cual ni siquiera puede ser concebido, pues es solo basándose en alguna teoría que se pueden elegir las propiedades que nos interesan. Por esto hablar de mero empirismo o, hasta peor, de materialismo a este respecto no tiene sentido, aunque esto tampoco significa que, al revés, el experimento pueda reducirse a la teoría, como pretende el anti-realismo epistemológico moderno (Musso. cap. 8). Al contrario, exactamente la profunda e ineludible unidad de razón y experiencia es la característica esencial del método científico galileano, que a su vez es el motivo esencial de su importancia filosófica en nuestro mundo moderno, que, como veremos, ha sido construido exactamente sobre el rechazo explícito de dicha unidad. Por fin, esto es también lo que hace que los experimentos sean repetibles, lo que es necesario para el control intersubjetivo, en que se basa la objetividad de todo conocimiento. En efecto, en nuestro mundo ninguna situación nunca se repite exactamente idéntica: luego, si fuese necesario considerar todos los factores, se debería concluir que ningún experimento es nunca realmente repetible, mientras si hacemos referencia solo a "algunas propiedades" la repetitividad es posible. ${ }^{5}$

3) El tercer punto del método galileano es el uso de la matemática para escribir las leyes naturales que el experimento debe verificar, como Galileo afirma en este famosísimo pasaje del Ensayador:

La filosofía natural [es decir, la ciencia] está escrita en ese enorme libro que continuamente queda abierto delante de nuestros ojos (yo digo el universo), pero no se la puede entender si antes no se aprende a entender la lengua, y conocer las letras, en las cuales está escrito. Eso está escrito en lenguaje matemático, y las letras son triángulos, círculos, y otras figuras geométricas, sin los cuales es humanamente imposible entender una sola palabra; sin estos sería como vagar sin meta por un oscuro laberinto. [Galileo 1623: 232]

4 Hay que decir que Galileo casi nunca usa el término "experimento" (solo 3 veces en toda su obra: dos en El ensayador y una en los Discursos y demostraciones matemáticas sobre dos nuevas ciencias), sino, según la costumbre de su tiempo, el término "experiencia". Sin embargo, en base al contexto se entiende claramente que está hablando del experimento en el sentido moderno del término. En cambio, esto será causa de cierta ambigüedad en el caso de Descartes.

5 En efecto, muchos objetaron a Galileo que estudiando propiedades aisladas se alteraba la realidad y hoy sabemos que en parte tenían razón, pues esto es exactamente lo que pasa en los sistemas caóticos (Musso, 2011. cap. 7): afortunadamente, el mundo no está hecho todo así, pues en este caso la ciencia sería imposible, pero Galileo esto no lo sabía, solo podía esperarlo. iSe necesitaba realmente una gran fe en la inteligibilidad de lo real para proponer un método de este tipo! 
Sin embargo, también la matemática, para poderse aplicar al estudio de la naturaleza, ${ }^{6}$ necesita un objeto definido de manera precisa y no ambigua: por ejemplo, las leyes del movimiento solo describen la trayectoria de un cuerpo, no su color o su temperatura. Luego, también este principio para funcionar tiene que limitarse al estudio de "algunas propiedades" y por lo tanto se basa en el primero.

4) Por fin, Galileo rechaza el principio de autoridad, pero no porque fuese un anarquista o un rebelde, como hoy es de moda pensar, sino solo porque para él en la ciencia hay una autoridad superior a la humana, que todos pueden interrogar a través de la matemática y el experimento, que es como una pregunta hecha a la naturaleza y, a través de esa, últimamente a Dios mismo, que es su Creador. Y como la matemática y el experimento a su vez se basan en el primer principio, entonces también este cuarto principio depende del primero, que por tanto es realmente $e l$ principio fundamental del método galileano.

Pero cuidado con entender correctamente lo que hemos recién dicho. No es que Galileo pensaba que el científico nunca debe fiarse de ninguno y siempre debe verificar por su cuenta todo lo que se le dice (como en cambio lo pensaba Descartes: Musso,2011. cap. 2), lo que volvería imposible tanto la enseñanza como el progreso científico: solo quería decir que, de no haber un acuerdo entre los científicos acerca de un cualquier asunto, para establecer quién tiene la razón siempre es el experimento que tiene la última palabra. Luego, en la práctica tampoco la ciencia puede prescindir del método del conocimiento por fe (que continuamente usamos en nuestra vida de cada día y que consiste en creer algo no porque lo hemos visto personalmente, sino en base a lo que dice un testigo fiable) y luego tampoco del principio de autoridad. Al contrario, pues, como recién acabamos de decir, dicho principio es la base de toda enseñanza, eso se vuelve cada vez más importante y crucial con el incremento del número, de la extensión y de la profundidad de nuestros conocimientos. Como lo decía el célebre lema de le Escuela de Chartres, «somos enanos a los hombros de gigantes», o sea, es solo porque podemos aprender de los que nos precedieron que podemos saber más que ellos, del cual derivan las dos características más peculiares de la ciencia: el ser por su naturaleza un trabajo abierto, ya que progresa continuamente, y el ser por su naturaleza un trabajo común, ya que el progreso no depende del hecho de que los que vienen después son más inteligentes de sus predecesores, sino del hecho de que, exactamente, pueden aprender de ellos todo lo que han descubierto y luego ir más allá. Por tanto, en la ciencia hay como una tensión entre el aspecto teórico y el práctico, que es inevitable y que cada científico tiene que manejar lo mejor que puede, intentando entender cuándo es oportuno fiarse y cuándo, en cambio, es mejor controlar personalmente. Es interesante notar que en todo esto juega un papel importante no solo la experiencia profesional, sino también la humana, porque la decisión de fiarse no se basa solo en un juicio técnico sobre la preparación científica del otro, sino también en un juicio sobre su fiabilidad como persona, o sea, en palabras simples, que no quiere estafarme

6 Es verdad que no todas las ciencias son enteramente matematizábles (se piense por ejemplo en la biología y más aun en las ciencias sociales), sin embargo, para hacer ciencia siempre se necesita por lo menos hacer referencia a algo medible. Además, siempre se necesitan unos instrumentos, que solo se pueden construir basándose en las ciencias más matematizadas: luego, en este sentido la tesis de Galileo sigue siendo verdad no solo para la física, sino para cualquier ciencia. 
(método de la certeza moral): luego, al fin y al cabo, en la realidad concreta, incluso el método científico, por más objetivo e impersonal que sea, puede prescindir completamente del factor humano.

\section{El pluralismo metodológico galileano}

Además, cabe subrayar que todo esto, como Galileo dice claramente en el texto de Las manchas solares que hemos recién citado y también en muchos otros, vale solo en el caso "de las sustancias naturales", o sea de los "cuerpos". Es decir, para Galileo, diferentemente a los antiguos, ${ }^{7}$ no hay un método único del conocimiento que va bien para todo: el método depende del objeto, luego objetos diferentes piden métodos diferentes (pluralismo metodológico, no reduccionismo).

En particular, la ciencia nace de una precisa y consciente autolimitación metodológica a los aspectos medibles de la realidad: luego, el reconocimiento de la legitimidad, más bien, incluso de la necesitad de la existencia de otras formas de conocimiento es un factor constitutivo de la ciencia misma y no algo que se le ha intentado imponerle indebidamente desde el exterior en un momento sucesivo. Y, de hecho, Galileo, pese a que nunca desarrolló una reflexión sistemática sobre las formas de conocimiento diferentes a la científica, que era la única que le interesaba y de la cual quería ocuparse, de todos modos, reconoció explícitamente el valor de dos otras formas de conocimiento además de la ciencia experimental (y, obviamente, de la matemática): la teología, basada en la Revelación divina, y el arte, basada en la capacidad de ensimismación en los sentimientos de los demás.

Luego, el mismo método científico, si bien entendido, es el mejor anticuerpo contra el cientificismo, sin ninguna necesidad de negar el valor cognoscitivo de la ciencia, como en cambio lo hace la epistemología moderna y como, lamentablemente, lo piensan también muchísimos filósofos (Musso, 2011. cap. 8).

Por fin, cabe subrayar que el método galileano, como hemos visto, es un conjunto de principios que siempre piden la interpretación creativa del científico y no un conjunto de reglas que solo hay que aprender y luego aplicar mecánicamente. Como se ha comprobado durante estos 4 siglos de ciencia, dichos principios son precisos lo suficiente como para indicar el camino bueno y descartar los malos, pero son también flexibles lo suficiente como para adaptarse a la constante evolución de la ciencia: y esto es sin duda uno de los mayores secretos de su éxito.

\section{El nacimiento de la ciencia: una cuestión cultural}

En fin, podemos preguntarnos: ¿por qué la ciencia nació en un preciso lugar y en un preciso tiempo, es decir, en la Italia del Renacimiento, al principio del siglo XVII?

Muchos historiadores dicen que dependió del progreso tecnológico y matemático que aconteció entonces. Sin embargo, la tecnología no jugó un papel determinante en los descubrimientos de Galileo (cf. Koestler, 1959; Jaki, 1978).

7 Es verdad que ttambién para Aristóteles existían diferencias entre los métodos con los cuales se podían conocer los principios de las varias ciencias y los niveles de certeza a los cuales podían llegar. Sin embargo, el esquema de fondo siempre era el mismo: en primer lugar, se debía establecer los principios básicos, para después deducir de esos todos los detalles. 
Solo su telescopio era un instrumento tecnológico, pero bastante simple. En cambio, por sus descubrimientos más fundamentales, los de las leyes del movimiento de los cuerpos, Galileo usó cuerdas, bolas, planos inclinados, relojes de agua, etcétera, es decir, todas cosas que siempre habían existido en cualquier civilización. Además, también su matemática era muy simple (una matemática realmente compleja empezó a necesitarse solo con Newton). Y entonces, ¿por qué la ciencia nació en Italia?

Si no fue por una cuestión tecnológica ni matemática, yo creo que fue por una cuestión cultural. Y estos fueron, en mi juicio, (cf. Foster 1934; Jaki 1978; Giussani, 1985; Hodgson, 2002; Bersanelli y Gargantini, 2003; Bersanello, 2016) los 3 factores culturales relevantes para el nacimiento de la ciencia moderna:

1) La fe griega y cristiana en un orden racional del mundo, que para ambas era un cosmos y no un caos, como en cambio era (y sigue siendo) en todas las religiones panteístas o animistas.

2) El redescubrimiento del platonismo $y$ de los textos de los matemáticos griegos, guardados y desarrollados por los árabes, aunque, más que un concreto instrumento de trabajo (como hemos dicho, la matemática usada por Galileo era muy simple), fueron sobre todo un estímulo cultural, en el sentido de difundir la idea de que la naturaleza tiene una estructura matemática.

3) Por fin y sobre todo, la fe cristiana en la creación del mundo, de la cual derivan:

3a) La contingencia del mundo. En efecto, la fe en la creación nos dice que el mundo es como es porque Dios así lo quiere, pero habría también podido ser diferente: ahora bien, esto es exactamente el fundamento metafísico último de la inversión metodológica galileana, ya que está claro que si el mundo es contingente, luego no hay principios necesarios de la naturaleza que, como tales, se puedan buscar solo por medio de la pura razón, como en lógica, matemática o metafísica, por lo que es necesario empezar de la experiencia y de los aspectos particulares que por su medio podemos conocer.

3b) La positividad de todas las cosas. Era realmente necesaria una fe cierta en la positividad y el valor de todo lo que existe para decidir que vale la pena estudiar no solo las cosas celestes, sino también las de nuestro mundo terrestre, que para los griegos era el reino de la imperfección, y sobre todo para entender que, para este fin, además del razonamiento, se necesita el trabajo manual, que para los griegos era una cosa de esclavos. Al contrario, para el cristianismo todo tiene el mismo valor porque todo ha sido creado por el mismo Dios, mientras que el trabajo manual es incluso un camino privilegiado hacia la santidad, considerando que Dios mismo quiso hacerse no solo hombre, sino artesano, siendo llamado comúnmente "el hijo del carpintero".

De lo anterior deriva una actitud que lleva un nombre muy bien conocido: humildad. Pero que no es, como muchas veces se dice, el sentimiento de cuán somos pequeños (que solo es depresivo), no es el sentimiento de cuán grande es la realidad, pero que, al mismo tiempo, 
misteriosamente pero realmente, es "para nosotros": porque esto sí, es lo que hace mover al hombre para conocerla.

En este sentido la verdadera humildad coincide de hecho con el sentido del misterio, que todos los científicos siempre han reconocido como esencial para su trabajo, empezando por el más grande de todos, Albert Einstein, que en un texto justamente famoso afirmó:

La más bella y profunda emoción que podemos probar es el sentido del misterio. En él se encuentra la semilla de todo arte y de toda ciencia verdadera. El hombre para el cual no resulta familiar el sentimiento del misterio, que ha perdido la facultad de maravillarse y humillarse ante la creación, es como un hombre muerto, o al menos ciego. (Einstein 1934: 37-38)

Claramente todo esto no significa que para ser buenos científicos sea necesario ser cristianos o de todos modos pertenecer a la civilización occidental, lo que sería una tontería absoluta, sino solo que del encuentro entre el cristianismo y la parte mejor de la cultura griega nació cierto clima cultural particularmente favorable para la ciencia, cuya actual difusión global es una prueba de la universalidad de nuestros valores, que hoy en día está tan de moda criticar.

Del mismo modo, no es nada casual que la ciencia ha nacido en el momento del máximo florecimiento del arte y de la cultura humanista. En efecto el método galileano es fundamental para volver rigurosas y verificables nuestras teorías, pero no existe un método para crear nuevas ideas: a este propósito lo único que puede ayudar es vivir en un contexto que educa lo más posible a mirar a la realidad en la totalidad de sus factores y a apreciar todo su fascino y belleza. Por ello descuidar las humanidades en nombre de un presunto "realismo" (como hoy lamentablemente es de moda) a la larga finaliza con hacerle daño también a la ciencia, como siempre lo dice también la gran física italiana Fabiola Gianotti, directora del CERN y descubridora del celebérrimo bosón de Higgs.

\section{Referencias bibliográficas}

Bersanelli, M.(2016). Il grande spettacolo del cielo. Otto visioni dell'universo dall'antichità ai giorni nostri, Sperling \& Kupfer, Milano. Traducción al castellano: El gran espectáculo del cielo. Ocho visiones del Universo desde la antigüedad a nuestros dias. Madrid: Ediciones Encuentro, 2018.

Bersanelli, M. y Gargantini,M. (Curadores). (2003). Solo lo stupore conosce, Rizzoli, Milano. Traducción al castellano: Solo el asombro conoce. La aventura de la investigación científica. Madrid: Ediciones Encuentro, 2006

Einstein, A. (1934). Mein Welbild. Asterdam: Querido

Einstein, A. (1938). The Evolution of Physics. The Growth of Ideas from Early Concepts to Relativity and Quanta. New York: Simon \& Schuster

Galilei, G. (1890-1909). Edizione Nazionale delle Opere di Galileo Galilei. Firenze: Giunti Barber

Galilei, G. (1613). Istoria e dimostrazioni intorno alle macchie solari e loro accidenti. Roma: Giacomo Mascardi. En Opere, vol. V, pp. 72-249.

Galilei, G. (1623). Il Saggiatore. Roma: Giacomo Mascardi. En Opere, vol. VI, pp. 199-372 
Galilei, G. (1632). Dialogo sopra i due massimi sistemi del mondo, tolemaico e copernicano. Firenze: Giovanni Battista Landini. En Opere, vol. VII, pp. 23-546.

Galilei, G. (1638). Discorsi e dimostrazioni matematiche sopra due nuove scienze. Leida: Elsevier. En Opere, vol. VIII, pp. 41-362.

Giussani, L.(1985). La coscienza religiosa nell'uomo moderno. Milano: Jaca Book. Traducción al castellano: La conciencia religiosa en el hombre moderno. Madrid: Encuentro, 1990

Hilbert, D.(1899). Grundlagen der Geometrie. Stuttgart: Teubner.
Hodgson, P.(2002). Christianity and science. Johannesburg: St. Augustine College.

Jaki, L.(1978). The Road of Science and the Ways to God. Chicago: Chicago University Press.

Koestler, A.(1959). The sleepwalkers. A history of man's changing vision of the universe. London: Hutchinson \& Co.

Musso, P. (2011). La scienza e l'idea di ragione. Scienza, filosofia e religione da Galileo ai buchi neri e oltre. Milano - Udine: Mimesis.

Recepción: 12-03-2019

Aceptación: 15-05-2019 